\title{
Los lectores expertos aprovechan mejor las anáforas: un estudio sobre la desventaja de la repetición nominal en la comprensión de textos
}

\author{
Amy Shapiro ${ }^{1}$ y Amy Milkes ${ }^{2}$ \\ ${ }^{1}$ Departamento de Psicología, Universidad de Massachusetts, North Dartmouth \\ ${ }^{2}$ Clínica Infantil Nemours, Jacksonville, Florida
}

ashapiro@umassd.edu 


\section{Resumen}

Introducción. La desventaja de la repetición nominal se refiere a la interferencia que experimentan los lectores cuando los pronombres se sustituyen por nombres repetidos $\left(^{*}\right)$. Se ha demostrado que la eliminación de referentes anafóricos disminuye la velocidad lectora (Gordon, Grosz, y Gilliom, 1993), pero no la comprensión. Este estudio quiso explorar si la desventaja de la repetición nominal sería evidente en postests de comprensión lectora. Ya que los buenos lectores aprovechan las pistas textuales de forma diferente que los malos lectores, también investigamos si los lectores buenos y malos estarían afectados por la ausencia de referencia anafórica de forma diferencial.

Método. Cada uno de los sujetos leyó dos textos, uno con pronombres y uno con nombres repetidos, y pasó los postests de comprensión.

Resultados. Los análisis de regresión mostraron que la desventaja de repetición nominal sí afecta la comprensión, pero sólo para los lectores muy expertos, lo cual sugiere que los lectores más expertos pudieron aprovechar mejor el uso de pronombres como pistas de la coherencia local. De forma coherente con el modelo construcción-integración de la comprensión de textos (Kintsch, 1988), este efecto se encontró en medidas de la adquisición de hechos, pero no la comprensión profunda.

Discusión. Estos resultados sugieren que la capacidad de aprovechar las anáforas como pistas para crear coherencia local de textos es un factor en la habilidad lectora. El que no se detectara el efecto de la repetición nominal sobre la comprensión en los estudios anteriores puede deberse a la brevedad de los pasajes de prueba utilizados en investigaciones previas.

* Nota de la Traductora: Al leer este artículo en español, hay que tener en cuenta que el estudio fue realizado en el idioma inglés, donde no se permite la omisión de un sujeto gramatical explícito en las oraciones. Es decir, que es obligatorio que el sujeto se exprese, o con un sustantivo, o con un pronombre. La repetición nominal en español podría consistir en repetir el sujeto de la oración anterior, donde normalmente no haría falta nombrar el sujeto en absoluto.

Palabras Clave: Anáfora, Modelo Construcción-Integración, Pronombres, Desventaja de Repetición Nominal, Coherencia de textos, Comprensión de textos. 


\section{Introducción}

La desventaja de la repetición nominal se refiere a un aumento en el tiempo de lectura que experimentan los lectores cuando los pronombres se sustituyen por nombres repetidos en las oraciones (Gordon, Grosz, y Gilliom, 1993). Este hallazgo es notable porque ofrece perspectiva sobre un aspecto importante de los procesos lectores. En concreto, apunta al uso de pistas de referencia en los lectores mientras descodifican las oraciones. Hasta la fecha, la desventaja de la repetición nominal sólo se ha estudiado en el contexto de párrafos breves y aislados, y no con textos completos. Además, se demostró que el efecto influyó sólo a la velocidad lectora y no a la comprensión ni a las medidas de aprendizaje. El propósito general de este estudio fue explorar la desventaja de la repetición nominal como factor en la comprensión de textos, ya que un gran número de trabajos han demostrado la relación de las características de los lectores con su aprendizaje. También exploramos la interacción entre el uso de pronombres y algunas características de los lectores. Específicamente, este trabajo examina la interacción entre la habilidad lectora, los conocimientos previos, y la presencia de pronombres o de nombres repetidos, por un lado, y la memoria y la comprensión de textos, por otro. Buscando la motivación para la selección de características de lectores como variables en este experimento, repasamos abajo varios trabajos anteriores. Este apartado lo sigue una discusión sobre la desventaja de la repetición nominal.

\section{Características de los lectores y de los textos que afectan la comprensión}

Tanto los conocimientos previos y la habilidad de comprensión lectora tienen mucha relación con la lectura y el aprendizaje a partir de textos. Está claramente demostrado en la investigación sobre comprensión de textos que los conocimientos previos en cierto dominio facilitan el proceso de comprensión (Recht y Leslie, 1988; Means y Voss, 1985; Spilich, Vesonder, Chiesi y Voss, 1979; Chiesi, Spilich y Voss, 1979). De hecho, Dochy, Segers y Buehl (1999) revisaron 183 libros, artículos y trabajos sobre conocimientos previos y encontraron que el 91.5\% informó sobre efectos positivos de los conocimientos previos sobre el rendimiento. Cuando los lectores cuántos más conocimientos tienen del tema del que leen, más pueden retener y recuperar la información del texto.

Por supuesto, la comprensión lectora también influye en cuánta información se aprende y/o se retiene al leer un texto (Laing y Kamhi, 2002; Long y Chong, 2001; Perfetti, 1989; Schmidt, Rozendal, y Greenman, 2002; Voss y Silfies, 1996). Perfetti (1989) argumentó que 
la lectura es una capacidad generalizada, que es independiente de los conocimientos. Aunque sugiere que tanto el conocimiento como la habilidad lectora contribuyen al proceso del aprendendizaje a partir de textos, ha mostrado que, con niveles de conocimiento iguales, los individuos que son lectores expertos podrán comprender y recordar mejor la información de textos, que los que son malos lectores.

Otro factor que afecta la comprensión del texto es una característica del texto en sí: la coherencia. La coherencia, en general, se refiere al grado de claridad con la que se relacionan las ideas dentro de un texto. Varios investigadores han demostrado los efectos de mayor coherencia de textos sobre la comprensión (Beck, McKeown, Sinatra, y Loxterman, 1991; Britton y Gulgoz, 1991; McNamara, Kintsch, Songer, y Kintsch, 1996; Voss y Silfies, 1996). Por ejemplo, Britton y Gulgoz (1991) adquirieron un texto desconocido sobre la Guerra de Vietnam, el cual tenía lagunas causales entre ideas, y lo revisaron para que las ideas y las oraciones se enlazaran más explícitamente. En otras palabras, incrementaron la coherencia local del texto. Encontraron que los alumnos universitarios que leyeron la versión modificada (coherente) recordaron más información, a un nivel significativo, en una prueba de libre memoria y contestaron correctamente a más preguntas de inferencia, a un nivel significativo, que los que leyeron la versión original.

Apoyándose en esta investigación, parece lógico suponer que hacer más fácil y más coherente a un texto favorecería la comprensión de todos los lectores. Sin embargo, las investigaciones han mostrado que la mejora de la coherencia de textos, la cual facilita su lectura, no es provechosa para todos los lectores (McNamara et al., 1996; McNamara y Kintsch, 1996; Voss y Silfies, 1996). McNamara et al. (1996) encontraron que los conocimientos previos se relacionan con la coherencia de textos. Los investigadores utilizaron cuatro versiones de un examen de biología, cada uno tenía coherencia local alta o baja, y coherencia global alta o baja. Se diseñaron medidas que comprobarían tanto la retención de hechos como el aprendizaje profundo de sus temas. Encontraron que en la prueba de memoria libre, no se relacionaron los conocimientos previos con el rendimiento. Sin embargo, en la resolución de problemas, los lectores con mayores conocimientos rindieron mejor con el texto de baja coherencia, y los lectores con menores conocimientos rindieron mejor con el texto de alta coherencia. McNamara y Kintsch (1996) reprodujeron los hallazgos de McNamara et al. (1996) con un examen de historia y una muestra de adultos. 
El trabajo de McNamara et al. (1996) está fundado en el modelo construcciónintegración de comprensión de textos de Kintsch $(1988 ; 1994)$. Kintsch distingue entre simplemente recordar un texto y realmente aprender de él. Una representación "base textual" (textbase) es lo suficiente para facilitar la memoria, y en algunos casos, el resumen de un texto. Sin embargo, un "modelo de situación" (situation model) permite que el lector tenga una comprensión más profunda del texto y que realmente aprenda a partir de él. Una base textual se forma cuando el lector crea una representación mental del material del texto en sí. Un modelo de situación, sin embargo, se forma cuando la base textual se integra con los conocimientos previos, lo cual resulta en una comprensión del tema más profunda y más compleja. McNamara et al. (1996) interpretan sus resultados como indicación de que los lectores de mayores conocimientos aprenden mejor a partir de textos menos coherentes porque les obligan a hacer inferencias y a aplicar activamente sus conocimientos previos a la información que van leyendo. En otras palabras: les ayudan a elaborar un modelo de situación. Sin embargo, los lectores con menos conocimientos previos se benefician de los textos de alta coherencia. El contenido textual añadido es útil porque no tienen los conocimientos para poder hacer inferencias sobre lo que no está en el texto. Estos lectores se benefician del texto ampliado porque es plenamente coherente y rico en información, así que les permite formar una sólida representación base textual.

Voss y Silfies (1996) exploraron los tres atributos de textos y de lectores que se tratan arriba (habilidad lectora, conocimientos previos, y contenido/coherencia del texto). Los investigadores elaboraron dos juegos de exámenes de historia ficticios. Cada juego incluía una versión ampliada y una versión no ampliada. La versión ampliada estaba plentamente desarrollada, con relaciones causales explícitas entre los acontecimientos y las ideas. La versión no ampliada no estaba bien desarrollada; simplemente describía los acontecimientos sin explicar su relación entre sí o cuál era el resultado final de la historia. Los investigadores proponían la hipótesis basada en el modelo de comprensión de textos de Kintsch (1988): la elaboración de una representación base textual estaría relacionada con la habilidad lectora y la elaboración de un modelo de situación estaría relacionada con los conocimientos previos. Predijeron además que los conocimientos previos y la habilidad lectora influirían en la lectura de forma diferencial, según el contenido del texto. Esto es precisamente lo que encontraron. Los conocimientos previos tenían correlación significativa con el resultado del aprendizaje cuando se leía el texto no ampliado y la habilidad lectora no la tenía; la habilidad lectora tenía 
correlación significativa con el resultado del aprendizaje cuando se leía el texto ampliado y los conocimientos previos no la tenían.

Voss y Silfies (1996) explicaron que cuando se lee un texto no ampliado, aquellos lectores con conocimientos previos puede llenar los huecos (hacer más coherente el texto), así pueden elaborar un modelo de situación y comprender plenamente el texto. Los lectores de mayores conocimientos no aprenden tanto de los textos de alta coherencia porque se les ofrece toda la información. Por lo tanto, no tienen motivo de relacionar la información del texto con lo que ya saben, o formar un modelo de situación. Los lectores de menores conocimientos no aprenden tanto de los textos no ampliados porque las inferencias son necesarias para la comprensión. No pueden hacer inferencias porque no tienen conocimientos previos en los cuales basarlas. Por otro lado, cuando el texto está plenamente desarrollado, los conocimientos previos no son necesarios para la comprensión porque toda la información está incluida en el texto. Además, la habilidad lectora es un factor en la comprensión de textos ampliados porque incluyen más información y son más difíciles de leer que los textos menos desarrollados.

En resumen, hay bastante evidencia que demuestra que la habilidad y los conocimientos previos de los lectores están relacionados con la comprensión, tal como lo está la coherencia del texto. A la luz de estos resultados, es importante estudiar la comprensión de textos dentro del contexto de estas variables.

\section{La Desventaja de la Repetición Nominal}

Gordon et al. (1993) llevaron a cabo una serie de experimentos de lectura en los cuales les dieron a los alumnos bloques individuales de varias oraciones, manipulando la presencia de los pronombres y su posición en la oración, y midieron tiempo de lectura y comprensión para cada grupo de frases. Encontraron que, bajo ciertas condiciones, el uso de nombres repetidos dió tiempos de lectura más lentos, comparado con oraciones iguales, pero que usaban pronombres. Gordon et al. (1993) se refieren a este efecto como la desventaja de la repetición nominal. Curiosamente, la desventaja de la repetición nominal sólo ocurría cuando el nombre estaba en una posición importante, por ejemplo como sujeto de la oración, y no cuando el nombre repetido estaba en una posición menos importante, por ejemplo el objeto de la oración (Gordon et al., 1993; Yang et al., 1999). Además, la desventaja de la repetición nominal depende de la relación estructural entre las oraciones. Siempre que una oración seguía con un referente importante de la oración anterior, ocurría la desventaja de la repetición no- 
minal. Sin embargo, cuando la posición del referente importante se cambió entre las oraciones, la desventaja de la repetición nominal no ocurría (Gordon et al., 1993).

De importancia para nuestra investigación, Gordon et al. (1993) no encontraron ningún efecto de la manipulación de pronombres sobre la comprensión, sólo sobre el tiempo de lectura. Sin embargo, su medida de la comprensión consistía en enunciados aislados de tipo verdadero/falso, para grupos de 3-5 oraciones. ${ }^{1}$ Con tan pequeña cantidad de información para descodificar y recordar, sospechamos que, en este estudio, el efecto no significativo del uso de pronombres sobre el rendimiento en la prueba se deba al contenido reducido. De hecho, la disminución en velocidad lectora que se asocia con los nombres repetidos sí sugiere que los pronombres facilitan la comprensión.

La clase de coherencia que ofrecen los pronombres parece ser de naturaleza distinta que la coherencia de textos que hemos comentado más arriba (Britton y Gulgoz, 1991; McNamara et al., 1996). La coherencia que se manipulaba en la mayoría de los estudios tenía que ver con cláusulas o frases añadidas para explicar las relaciones. Nosotros nos referimos a la coherencia creada por las anáforas como coherencia referencial porque las anáforas aumentan la coherencia señalando las relaciones entre ideas, más que por ofrecer información real que explique las relaciones. En otras palabras, los pronombres ayudan a que los lectores enlacen las oraciones indicando un sujeto común, así relacionándolas conceptualmente. De hecho, el tiempo de lectura incrementado en la ausencia de los pronombres, tal como observó Gordon et al. (1993), sugiere que la comprensión de textos sin pronombres requiere algo de procesamiento añadido por parte de los lectores.

Las teorías sobre el aprendizaje a partir de textos nos sirven para explicar por qué la repetición nominal en un texto interferiría con el aprendizaje. Van Dijk (1980) propuso que la información aprendida está incorporada o almacenada en macro-estructuras. Su teoría sugiere que unidades de información son enlazadas para formar estas macro-estructuras que sirven tanto para organizar como para reducir la información compleja. Van Dijk argumenta que las macro-estructuras nos permiten formar "bloques" de información más grandes, que "tienen sus debidos significado y función" (p. 14). Además, sostiene que asignar una unidad macroestructural a una serie de hechos independientes (que él llama "unidades a nivel microestructural") define la relación temática entre ellos. Ya que los pronombres le indican al lec-

\footnotetext{
${ }^{1}$ El diseño de ese studio tenía como objetivo la comprobación de predicciones de la Teoría de Centering. Este trabajo no tiene que ver con la Teoría de Centering, sino con la exploración de los efectos de la coherencia creados por la referencia anafórica sobre el aprendizaje a partir de textos.
} 
tor que el sujeto de una oración es el mismo que el de una oración anterior, sirven para ayudar a los lectores a que relacionen la información entre las oraciones. En la terminología de Van Dijk, les indican a los lectores a que relacionen las unidades de información dentro de una macro-estructura común.

Kintsch $(1988$; 1994) amplió la teoría de van Dijk con su modelo construcción integración. Como mencionamos arriba, Kintsch propone que esta macro-estructura se forma en la memoria cuando la información contenida en un texto se almacena. Estas estructuras, que él llama bases textuales, reflejan la organización del texto y están construidas a partir de su contenido semántico. Por lo tanto, dentro del modelo construcción-integración, los pronombres pueden considerarse también una pista para los lectores, que las ideas entre las oraciones deben ser unidas dentro de una estructura común. El modelo construcción-integración también propone que otra clase de estructura, el llamado modelo de situación, también se puede formar durante la lectura. Los modelos de situación contienen la información de la base textual e información adicional de la memoria permanente. El modelo de situación, por tanto, se puede concebir como el almacén de nuestra comprensión más profunda del material escrito. Una forma de procesamiento más compleja es necesaria para la creación de un modelo de situación, pues la nueva información debe ser integrada con las experiencias y los conocimientos previos.

El modelo construcción-integración predice que la desventaja de la repetición nominal ocurriría en la construcción de la base textual (p.ej, información factual del texto en sí). Sin embargo, no predice diferencias en las medidas de modelo de situación (es decir, comprensión profunda) como resultado de nombres repetidos versus textos con pronombres, pues la referencia anafórica no les ayuda a los lectores a que relacionen el texto con los conocimientos previos. Los pronombres sólo ayudan al lector a que cree coherencia local dentro de un texto, y así facilita la construcción de la base textual.

Para comprobar estas predicciones, en este trabajo se presentó a los sujetos textos que contenían o bien nombres repetidos o bien pronombres. Los postests incluían medidas de conocimiento factual (respuesta breve) y de comprensión más profunda (redacción). Había tres hipótesis principales de este trabajo. La primera es que la desventaja de la repetición nominal sí ocurriría, mostrada por un rendimiento en el postest significativamente mejor para la condición de pronombres que para la condición de nombres repetidos. Nuestra segunda hipótesis era que habría una reacción entre la habilidad lectora y el uso de pronombres. Los bue- 
nos lectores aprovechan las pistas textuales de forma diferente que los malos lectores (Oakhill y Yuill, 1986). Por lo tanto, los lectores buenos y malos deben estar afectados de forma diferencial por la coherencia referencia. Consecuentemente, con el modelo construcciónintegración de la comprensión de textos (Kintsch, 1988), las dos hipótesis se afirman respecto al postest de conocimiento factual, pero no para el postest de redacción. Es decir, se espera que estas variables afecten a la construcción de bases textuales, pero no los modelos de situación. Nuestra tercera hipótesis era que los conocimientos previos no reaccionarían con el uso de pronombres, ya que un texto pobre en coherencia referencial, no se puede "arreglar" con conocimientos previos; nosotros vemos la coherencia referencial como una cuestión sólo de lectura y no de contenido. No obstante, sí predijimos que los conocimientos previos serían un factor importante por sí solos en la calidad de las redacciones de los sujetos. Dado que muchos trabajos anteriores han mostrado que los conocimientos previos predicen la comprensión más profunda de textos en los sujetos (Dochy, Segers y Buehl, 1999), esperamos encontrar que los conocimientos previos tuvieran una aportación importante a la calidad de sus redacciones.

\section{Método}

\section{Sujetos}

Los participantes fueron 57 estudiantes universitarios no licenciados de la Universidad de Massachusetts, los cuales participaron para cumplir un requisito de su asignatura de introducción a la psicología. Había, más o menos, el mismo número de hombres que de mujeres.

\section{Materiales}

Los materiales utilizados en este trabajo se basaron en creados por Voss y Silfies (1996). Ellos elaboraron dos textos, cada uno describía un país ficticio. Los utilizamos porque Voss y Silfies (1996) ya habían hecho equivalentes los dos textos, y nos interesaba un diseño entre-sujetos. Además, los postests que Voss y Silfies elaboraron para estos textos ya fueron utilizados con éxito en investigaciones sobre el procesamiento de textos. Un texto se llama "Anchad" y el otro "Padria", los títulos se corresponden con el nombre del país de que se trata cada texto. El texto Anchad, con pronombres, tenía 742 palabras, y con repetición nominal, tenía 802 palabras. Los textos Padria tenían 753 palabras y 778 palabras, respectivamente. Se refiere a ellos como textos "completos" porque son pasajes completos, compa- 
rados con los grupos de oraciones aisladas de estudios previos. En cada texto, se describe una serie de acontecimientos que conducen al estallido de una guerra.

Todos los participantes recibieron tanto un texto de Anchad como uno de Padria, uno de ellos hacía uso de pronombres, y el otro no. El orden fue equilibrado, en que la mitad de los participantes recibieron primero un texto de Anchad, y la mitad recibieron primero un texto de Padria. También fue equilibrado en que se presentaron a los subjetos las cuatro posibles combinaciones de versiones y de orden de los dos textos.

Para comprobar la desventaja de la repetición nominal, los textos Anchad y Padria fueron ligeramente modificados para que el mismo sujeto de la oración apareciera al principio de varias oraciones consecutivas. Esta modificación no afectó el contenido del texto, sólo cambió el orden de algunas palabras ya existentes en el texto. Una versión de cada texto tenía nombres de sujeto repetidos en las oraciones consecutivas, y otra versión utilizaba un nombre de sujeto en la primera oración, y un pronombre como subjeto en la oración siguiente, como queda ilustrado en este ejemplo:

\section{Oración inicial:}

John Lerner is the leader of the resistance movement. [John Lerner es el dirigente del movimiento de la resistencia.]

Oración 2 en la condición de nombres repetidos como sujeto:

John Lerner is a peasant whose parents were employed as crop laborers. [John Lerner es un campesino cuyos padres tenían empleo como trabajadores del campo.]

Oración 2 en la condición de pronombres:

$H e$ is a peasant whose parents were employed as crop laborers. [Es un campesino cuyos padres tenían empleo como trabajadores del campo.]

En ambos textos Anchad y Padria había 14 conjuntos de sujetos repetidos en oraciones consecutivas 
Procedimiento

Pruebas de pretest. Primero, los participantes pasaron la parte sobre comprensión lectora de la prueba Nelson-Denny de lectura. La Nelson-Denny es una prueba de la comprensión lectora validada y que ha sido utilizada en cientos de artículos publicados que están relacionados con la investigación sobre lectura. Se ha demostrado que predice acertadamente diversas medidas de rendimiento académico (Hawes, 1982; Wood, 1982; Zimmer, Glover, Ronning, y Petersen, 1979). Esta prueba tenía limitación de tiempo, pero a los sujetos se les permitió trabajar a su propio ritmo para todo lo posterior de esta investigación. Luego los participantes completaron un cuestionario demográfico y una prueba de Voss y Silfies (1996) de 20 preguntas, diseñado para medir sus conocimientos de la historia mundial.

Lectura y pruebas de postest. Se les decía a los participantes que leyeran con atención el primer texto, y que después responderían a preguntas sobre el mismo. Cuando terminaban de leer, recibían las preguntas de respuesta breve. A los participantes no se les permitía consultar el texto para contestar las preguntas. Después se les pedía que escribieran la redacción. Para esto se les permitía consultar el texto. Cuando terminaron, se les daba el segundo texto, y se repetía el procedimiento. El tiempo total para cumplir el estudio varía entre una hora y una hora con 45 minutos.

\section{Análisis estadístico}

Medidas dependientes. Las pruebas de respuesta breve para cada texto las elaboraron Voss y Silfies' (1996). Las respuestas para cada pregunta se encontraban en el mismo texto. De esta manera son medidas del conocimiento del sujeto de la información factual que ha retenido del texto en sí, o sea, una medida de la base textual.

A los sujetos también se les pidió redactar su respuesta a una pregunta sobre las causas subyacentes del conflicto que se describe en el texto. Se hizo la misma pregunta tanto para el texto de Padria como para el texto de Anchad. Estas preguntas les obligaron a los sujetos a que pensaran más allá de la información factual que se presenta en el texto, y que se pusieran a analizar las causas del conflicto. Las respuestas a esta pregunta reflejan la comprensión del texto más profunda de los sujetos, y así fueron utilizadas como medidas de elaboración de modelos de situación.

Puntuación de los datos. Se puntuaron las redacciones en una escala de 6 puntos, diseñada para reflejar la calidad del contenido de la redacción. La escala fue de 0 a 5 , véase la 
Tabla 1 para los criterios de puntuación concretos. Los investigadores puntuaron independientemente grupos de redacciones tanto sobre Anchad como sobre Padria. Para evitar efectos de fatiga mental, se puntuaba un máximo de cinco redacciones en una sola sesión. Puntuamos varias redacciones de Anchad y Padria como práctica, y después nos reunimos para hablar de las puntuaciones, lo que ayudó a perfeccionar la escala y hacer más precisa la puntuación. Como prueba final de la fiabilidad de nuestro método de puntuación, cada uno puntuamos 10 redacciones de Padria y 10 de Anchad independientemente. La fiabilidad de nuestra puntuaciones fue $r=.795$ para las redacciones de Anchad y $r=.742$ para las redacciones de Padria. Las preguntas de respuesta breve tenían cada una por respuesta una sola palabra o una sola frase. Cualquier respuesta que no correspondiera exactamente a lo que venía escrito en la hoja de respuestas se puntuó como incorrecta. La única excepción fue el uso de nombres singulares vs. plurales, o el tiempo verbal.

Tabla 1: Pautas utilizadas para normalizar la puntuación de las redacciones

\begin{tabular}{|c|l|}
\hline PUNTUACIÓN & CRITERIOS* \\
\hline 0 & $\begin{array}{l}\text { Ninguna respuesta para la pregunta; o bien, el contenido es incorrecto del todo, o es irrele- } \\
\text { vante del todo. }\end{array}$ \\
\hline 1 & $\begin{array}{l}\text { Responde a la pregunta, pero sólo dice generalidades. La respuesta puede mencionar algo } \\
\text { del texto, pero sólo hay muy poco que sea acertado y/o relevante. }\end{array}$ \\
\hline 2 & $\begin{array}{l}\text { La respuesta a la pregunta es algo relevante. En concreto, hay una mezcla de puntos acer- } \\
\text { tados y no acertados, relevantes y no relevantes. No se puede clasificar como "acerta- } \\
\text { do/relevante" en su mayor parte, ni "no acertado/no relevante" en su mayor parte. }\end{array}$ \\
\hline 3 & $\begin{array}{l}\text { La respuesta a la pregunta es relevante y acertada en su mayor parte (puede haber una } \\
\text { frase o dos que sean problemáticas). La respuesta se extrae mayormente del mismo texto, } \\
\text { pero sí tiene relación con la pregunta propuesta. Aunque se incluyen puntos relevantes en } \\
\text { la respuesta, hay poco o ningún intento de explicar cómo contribuyeron al conflicto. El } \\
\text { estudiante puede ofrecer una tesis/hipótesis como causa del conflicto, pero esta tesis ó (1) } \\
\text { no se apoya en los hechos incluidos en la redacción, ó (2) es incorrecta. }\end{array}$ \\
\hline 4 & $\begin{array}{l}\text { La respuesta a la pregunta es relevante y acertada en su mayor parte (puede haber una } \\
\text { frase o dos que sea problemática). La respuesta se extrae mayormente del mismo texto, } \\
\text { pero sí tiene relación con la pregunta propuesta. Además, el estudiante empieza a explicar } \\
\text { la relación entre diversos puntos como forma de acercarse a la raíz del problema. Sin } \\
\text { embargo, la explicación está limitada al texto o a ciertas explicaciones superficiales. }\end{array}$ \\
\hline 5 & $\begin{array}{l}\text { La respuesta a la pregunta es relevante y seria con bastante sustancia. Cierta perspicacia } \\
\text { está presente en la respuesta y se citan diversas causas. El estudiante se ha dedicado a } \\
\text { cierto análisis de las causas de los acontecimientos citados, más allá del texto en sí. }\end{array}$ \\
\hline
\end{tabular}

* No hacer ningún caso a cualquier referencia a la acción militar en sí, y lo que ocurriera después de la acción militar. Estas referencias no cuentan ni a favor ni en contra de la respuesta. En el texto de Padria, no hacer caso al bombardeo de Padria ni a los acontecimientos que lo siguen. En el texto de Anchad, no hacer caso a la concentración de tropas en las fronteras ni a los acontecimientos que lo siguen. 


\section{Resultados}

\section{Análisis de correlación}

Para comprobar la validez de nuestras medidas, se calcularon correlaciones entre las variables de conocimiento y de comprensión, y las variables dependientes. Ya que está demostrado que ambas medidas predicen los resultados de aprendizaje, lo mismo debe ser cierto, por lo general, en este caso. La tabla 2 expone estos resultados. Como predijimos, la puntuación de conocimiento histórico se correlacionó significativamente con el rendimiento en los postests tanto de respuestas breves como de redacción, y tanto en la condición de pronombres como en la condición de nombres repetidos. También se confirmó nuestra predicción que las puntuaciones de comprensión de textos guardarían correlación significativa con el rendimiento en la prueba postest, pues la comprensión lectora se correlacionó significativamente con el rendimiento en todas las medidas menos la medida de la redacción en su condición de pronombres.

Tabla 2: Correlaciones de Variables de Tipo Comprensión y de Tipo Conocimiento con Variables Dependientes.

\begin{tabular}{|c|c|c|c|c|}
\hline & \multicolumn{2}{|c|}{ PRONOMBRES } & \multicolumn{2}{|c|}{ NOMBRES REPETIDOS } \\
\hline Variables & $\begin{array}{c}\text { Respuesta } \\
\text { Breve }\end{array}$ & Redacción & Respuesta Breve & Redacción \\
\hline \multicolumn{5}{|l|}{ Tipo Conocimiento } \\
\hline Conocimiento de la Historia & $.41 * *$ & $.44 * *$ & $.53 * *$ & $.43 * *$ \\
\hline Interés en la Historia & .25 & .01 & $.32 *$ & .25 \\
\hline $\begin{array}{l}\text { Número de asignaturas sobre de His- } \\
\text { toria }\end{array}$ & .14 & .08 & .08 & .14 \\
\hline $\begin{array}{l}\text { Número de asignaturas sobre cien- } \\
\text { cias políticas }\end{array}$ & .01 & -.11 & -.10 & -.05 \\
\hline $\begin{array}{l}\text { Interés en los acontecimientos actua- } \\
\text { les }\end{array}$ & .07 & -.11 & .03 & .15 \\
\hline \multicolumn{5}{|l|}{ Tipo Comprensión } \\
\hline Comprensión Lectora & $.44 * *$ & .26 & $.36^{* *}$ & $.40 * *$ \\
\hline Velocidad Lectora & .18 & $.29 *$ & .09 & .00 \\
\hline Media de Notas Académicas & .04 & $.27 *$ & .01 & .12 \\
\hline $\begin{array}{l}\text { Puntuación Verbal en la prueba } \\
\text { Scholastic Aptitude Test (SAT) }\end{array}$ & $.34 *$ & .27 & $.44 * *$ & $.38 * *$ \\
\hline
\end{tabular}

Nota. $* \underline{p}<.05 . * * \mathrm{p}<.01$ Comparaciones Simples 
La predicción de un efecto principal de la condición de los pronombres no fue apoyada por los datos. Las puntuaciones medias para las respuestas breves para condiciones de nombres repetidos y de pronombres eran 4,79 y 4,78, respectivamente. Las puntuaciones medias para las redacciones para estas condiciones eran 2.79 y 2.84 , respectivamente. En ambos casos las diferencias no son significativos, $\mathrm{t}(57)<1$. En contra de nuestra predicción, reprodujimos el hallazgo original de Gordon et al. (1993). Ellos informaron sólo de un aumento en el tiempo de lectura, asociado con la repetición nominal, y no de un efecto principal de manipulación de pronombres sobre su medida de comprensión. El siguiente apartado examina nuestra segunda hipótesis, referida a que la desventaja de la repetición nominal está presente cuando se la considera dentro del contexto de la habilidad lectora. Puede ser que la manipulación de pronombres sólo afecte a la comprensión como relación con la habilidad lectora.

\section{Análisis de Regresión}

Se efectuaron análisis de regresión para examinar la aportación de conocimientos previos y de habilidad lectora en la memoria y comprensión de textos, tanto para los textos de nombres repetidos como para los de pronombres. No fue posible usar todas variables de tipo conocimiento ni de tipo comprensión en estos análisis, porque el número de participantes disponibles para este trabajo no sostendría este gran número de variables independientes. Por lo tanto, la puntuación de las pruebas pretest de comprensión lectora y de conocimiento histórico se utilizaron como medidas de la habilidad lectora y de los conocimientos previos en un análisis de regresión múltiple. Éstas fueron seleccionadas porque son las medidas más directas de la habilidad lectora y de los conocimientos históricos que se incluyeron en este trabajo.

Se llevaron a cabo dos conjuntos de regresiones múltiples con medidas repetidas. Cada análisis incluía dos factores independientes continuos de grupo (puntuación de lectura y de historia) y un factor de medidas repetidas (pronombres vs. nombres repetidos). Un análisis se efectuó con las puntuaciones de repuestas breves (número de ítems correctos) como variable dependiente, y uno con puntuaciones de las redacciones como variable dependiente. Para hacer una regresión múltiple de medidas repetidas, es necesario hacer dos regresiones múltiples por separado: uno en el que se toma la media de la variable dependiente a lo largo de las condiciones de medidas repetidas (pronombres y nombres repetidos), y un segundo en el que la medida repetida sirve como variable de imitación (Cohen y Cohen, 1983). Se examinaron los datos según las suposiciones de la regresión con éxito, con la excepción de un extraviado en los datos de las respuestas breves (sujeto 7), este dato se substrajo del análisis de las res- 
puestas breves. Primero veremos el análisis de regresión que utilizó puntuaciones de las respuestas breves como variable dependiente, y después la regresión que utilizó puntuaciones de las redacciones como variable dependiente.

Preguntas de respuesta breve. Las puntuaciones de lectura y de historia juntas fueron significativas, $\mathrm{R}^{2}=.48, \underline{\mathrm{F}}(2,55)=25.36, \underline{\mathrm{p}}<.01$. Consideradas por separado, la puntuación de historia fue significativa $(\mathrm{sr}=.46, \underline{\mathrm{p}}<.01)$ y la puntuación de lectura también fue significativa $(\mathrm{sr}=.29, \mathrm{p}<.01) . \mathrm{El}$ factor de medidas repetidas no fue significativo, $\mathrm{R}^{2}=.00, \underline{\mathrm{F}}(1,55)$ $=.00$. Las interacciones en dos sentidos, consideradas en su totalidad, fueron significativas $\left(\mathrm{R}^{2}=.09, \underline{\mathrm{F}}(3,50)=2.84, \underline{\mathrm{p}}<.05\right)$. Consideradas por separado, la interacción de la lectura por medidas repetidas fue significativa $(\mathrm{sr}=.35, \underline{\mathrm{t}}(54)=2.76, \underline{\mathrm{p}}<.01)$, pero la de los conocimientos históricos por medidas repetidas no fue significativa. Por fin, la interacción en tres sentidos no fue significativa, $\mathrm{R}^{2}=.03, \underline{\mathrm{F}}(1,49)=1.50$.

Para determinar la dirección de los efectos en la interacción de lectura por medidas repetidas, primero se dividieron los sujetos entre tres grupos según su puntuación de comprensión de lectura. La media de las puntuaciones de lectura fue 22.3, con una desviación típica de 5.5. El grupo bajo se constituyó con aquellos quienes puntuaron con una desviación típica por debajo de la media; el grupo alto puntuó una desviación típica por encima de la media, y el grupo medio puntuó entre estos dos extremos. Luego se trazaron en un gráfico las medias de las puntuaciones de las respuestas breves en la condición de pronombres y en la condición de nombres repetidos para cada grupo de habilidad lectora (bajo, medio, y alto). Este gráfico se ve en la Figura 1.

Figura 1: Rendimiento en las respuestas breves dadas por lectores de habilidad baja, media, $y$ alta en las condiciones de nombres repetidos y de pronombres.

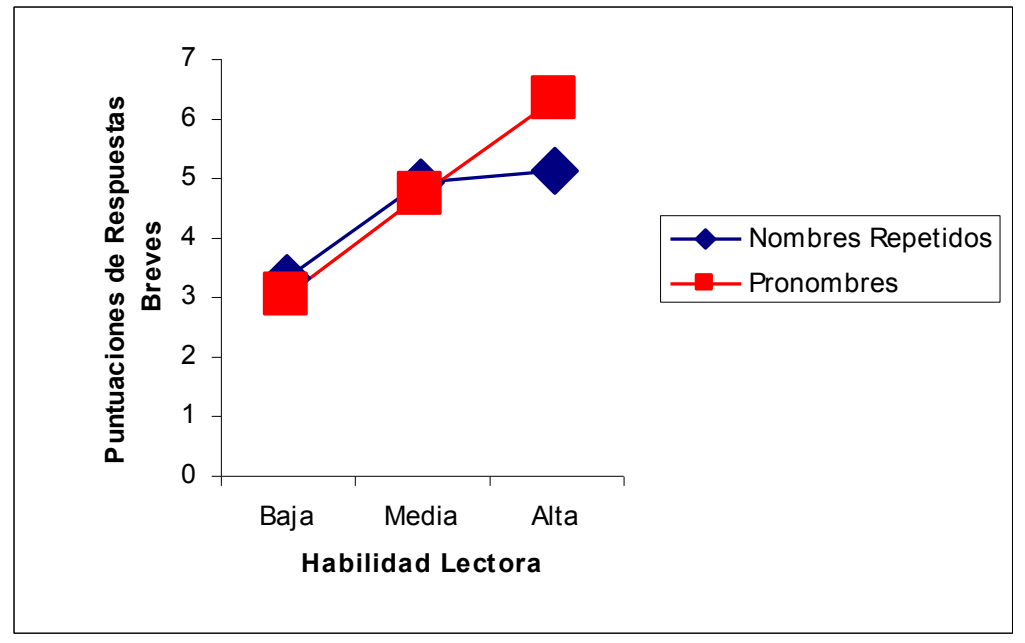


Preguntas de tipo redacción. Los resultados de la regresión múltiple mostraron que la puntuación de lectura y la puntuación de historia, introducidas juntas, fue un factor significativo $\left(\mathrm{R}^{2}=.32, \underline{\mathrm{F}}(2,55)=13.10, \underline{\mathrm{p}}<.01\right)$. Consideradas por separado, la puntuación de historia fue significativa $(\mathrm{sr}=.40, \underline{\mathrm{p}}<.01)$, pero la puntuación de lectura no lo fue. El factor de medidas repetidas no fue significativo, $\mathrm{R}^{2}=.001, \underline{\mathrm{F}}(1,55)=.07$. Ni las interacciones en dos sentidos, consideradas en su totalidad, ni la interacción en tres sentidos fueron significativos, $\mathrm{R}^{2}=.005, \underline{\mathrm{F}}(3,50)=.24$ y $\mathrm{R}^{2}=.008, \underline{\mathrm{F}}(1,49)=1.154$, respectivamente.

\section{Discusión}

Nuestra primera hipótesis era que el uso de pronombres sería un factor significativo e independiente para las regresiones de las respuestas breves, pero no para las regresiones de las redacciones, y que las puntuaciones en la condición de pronombres serían significativamente más altas que sus homólogos en la condición de nombres repetidos. Sin embargo, el factor de medidas repetidas no fue significativo por sí solo en ningún análisis. Este hallazgo nos enseña que, en contra de nuestra idea inicial, los nombres repetidos en general no perjudican a los lectores en cuanto a la comprensión, cuando se los considera como variable aislada. Este hallazgo, por lo tanto, reproduce los resultados de postest de memoria de Gordon et al. (1993), y refuta nuestra primera hipótesis.

No obstante, considerados dentro del contexto de habilidad lectora como variable, la desventaja de la repetición nominal era evidente, así apoyando nuestra segunda hipótesis. En la condición de pronombres, el rendimiento de los lectores con mayor habilidad lectora fue significativamente mejor que el de los otros participantes en las preguntas de respuesta breve. En la condición de nombres repetidos, sin embargo, el rendimiento de los participantes con mayor habilidad lectora fue más parecido al de sus homólogos menos expertos. Como predijimos, la interacción entre habilidad lectora y el uso de pronombres sólo ocurrió en el postest de respuesta breve.

La reacción significativa en la prueba de respuesta breve revela que la coherencia referencial es importante para la construcción de la base textual para lectores expertos. Este resultado se parece al de Voss y Silfies (1996), quienes encontraron que la habilidad lectora predecía el rendimiento en un postest factual después de leer un texto altamente coherente, pero no con un texto menos coherente. Igual que los resultados de este trabajo, su manipulación de coherencia afectó las pruebas de elaboración de base textual pero no del modelo de 
situación. El paralelismo entre los resultados de Voss y Silfies y los expuestos aquí sugiere que lo que llamamos coherencia referencial, conseguida por el uso de pronombres, afecta la comprensión de una manera similar a otras manipulaciones de coherencia de texto. En concreto, la coherencia referencial afecta a los lectores de una manera parecida a la agregación de frases o cláusulas que sirven para explicar las relaciones entre ideas.

Al principio de esta investigación, propusimos que Gordon et al. (1993) no observaron ningún efecto de su manipulación de pronombres sobre la comprensión, a causa de los párrafos cortos que utilizaron como estímulos. La ausencia de un efecto principal de manipulación de pronombres sobre las medidas de comprensión en este trabajo, sugiere que esta idea era incorrecta. En cambio, la interacción con la habilidad lectora sugiere que, para medidas de comprensión, la desventaja de la repetición nominal sólo existe para lectores expertos. Dado que Gordon et al. no probaron su medida de comprensión dentro del contexto de habilidad lectora, no podían detectar la desventaja de la repetición nominal para la comprensión. El trabajo de Gordon et al. sugiere que los lectores ocupan más tiempo leyendo un texto con nombres repetidos; nosotros ampliamos sus hallazgos, demostrando que sólo lectores expertos sufren una relativa disminución en su comprensión.

Una explicación probable para esta interacción entre la habilidad lectora y el uso de pronombres es que los lectores expertos aprovechan mejor las pistas, como los pronombres, para ayudarles en su comprensión. Como consecuencia, cuando los lectores expertos leen un texto con pronombres, se benefician de un uso eficaz de los pronombres como pistas. Sin embargo, cuando leen un texto con repetición nominal, falta esta herramienta útil y les afecta negativamente a la hora de descodificar el texto. Los individuos de habilidad lectora media o baja pueden tener más dificultad en el uso de pronombres como pistas para la comprensión, así que tienen el mismo rendimiento si leen un texto con pronombres o con repetición nominal. Esta explicación está apoyada por Oakhill y Yuill (1986), que estudiaron la capacidad de niños de habla inglesa de 7 y 8 años para comprender los pronombres. Les administraron una serie de cláusulas de dos oraciones y les pidieron que identificaran los referentes de los pronombres. Encontraron una mayor incidencia de errores por parte de los malos lectores, y sugieren que la dificultad en la interpretación y comprensión de pronombres puede contribuir a la dificultad experimentada por los malos lectores.

Como predijimos, no hubo contribución independiente del uso de pronombres sobre el rendimiento en las redacciones. Además, ninguna de las dos variables de sujeto interaccionó 
con la manipulación de pronombres en esta medida. Estos resultados indican que la comprensión más profunda que fue medida por las redacciones no fue afectada por la coherencia referencial. En cambio, de acuerdo con nuestra tercera hipótesis, sólo los conocimientos previos en sí mismos contribuyeron de forma independiente y significativa al rendimiento en las redacciones. Este patrón de resultados se explica por el modelo construcción-integración (Kintsch, 1988). Los pronombres sirven para ayudar a que los lectores creen coherencia local, $\mathrm{y}$, por tanto, representaciones de tipo base textual fuerte y acertada. Los pronombres no les ayudan a integrar la información con conocimientos previos, el proceso por el que se contruye un modelo de situación (comprensión más profunda). Ya que las redacciones fueron diseñadas para valorar la comprensión más profunda, se predijo el efecto no significativo de la manipulación de pronombres para esa medida. Por otro lado, tiene sentido entender que mayores conocimientos previos se asociaban con un mejor rendimiento en la prueba de redacciones. Cuánto más conocimiento tiene el sujeto, mejor podrá integrarlo con el contenido del texto y favorecer la comprensión. De hecho, los resultados de las redacciones están de acuerdo con otros presentados en varios trabajos anteriores (véase Dochy et al., 1999, para una revisión). Mientras la hipótesis 3 no sirve para conocer mejor el uso de pronombres en la comprensión de textos, sí tiene que ver con la validez de nuestros conocimientos previos y las medidas de comprensión profunda.

Hasta ahora, la desventaja de la repetición nominal, definida por Gordon et al. (1993), no se ha estudiado con pasajes de texto completos y naturales, ni conjuntamente con las características de los lectores. No encontramos evidencia para decir que la extensión de un texto esté relacionada con la presencia de la desventaja de la repetición nominal en la comprensión. Sin embargo, sí encontramos que la desventaja de la repetición nominal, de hecho, se aplica a las medidas de retención y aprendizaje a partir de textos, pero sólo para los lectores más expertos. Es más, el efecto diferencial de este tipo de coherencia sobre los lectores de distinta habilidad puede revelarnos un factor importante en la habilidad lectora. ¿Qué es lo que hace "buenos" a los buenos lectores? Los resultados de este trabajo sugieren que un factor es la capacidad para aprovechar eficazmente las pistas referenciales para crear coherencia local. Otros han demostrado que la coherencia local de un texto afecta la comprensión de forma significativa (Britton y Gulgoz, 1991). Los resultados aquí presentados sugieren que los lectores expertos crean esta clase de coherencia, en parte, con la ayuda de estas señales referenciales. 


\section{Referencias}

Beck, I.L., McKeown, M.G., Sinatra, G.M., y Loxterman, J.A. (1991). Revising social studies text from a text-processing perspective: Evidence of improved comprehensibility. Reading Research Quarterly, 27, 251-276.

Britton, B. K., y Gulgoz, S. (1991). Using Kintsch's computational model to improve instructional text: Effects of repairing inference calls on recall and cognitive structures. Journal of Educational Psychology, 83, 329-345.

Chiesi, H. L., Spilich, G. J., y Voss, J. F. (1979). Acquisition of domain-related information in relation to high and low domain knowledge. Journal of Verbal Learning and Verbal Behavior, 18, 257-273.

Cohen, J., y Cohen, P. (1983). Applied multiple regression/correlation analysis for the behavioral sciences (2nd ed.). Hillsdale, NJ: Erlbaum.

Dochy, F., Segers, M., y Buehl, M. M. (1999). The relation between assessment practices and outcomes of studies: The case of research on prior knowledge. Review of Educational Research, 69, 145-186.

Gordon, P. C., Grosz, B. J., y Gilliom, L. A. (1993). Pronouns, names, and the centering of attention in discourse. Cognitive Science, 17, 311-347.

Hawes, K. (1982). Correlations of scores on the Nelson-Denny Reading Test, Form E, and measures of academic aptitude, academic achievement, readers' attitude, and students' self-concept. Psychological-Reports, 51, 229-230.

Kintsch, W. (1988). The use of knowledge in discourse processing: A construction integration model. Psychological Review, 95, 163-182.

Kintsch, W. (1994). Text comprehension, memory, and learning. American Psychologist, 49, 294-303.

Laing, S. P., y Kamhi, A. G. (2002). The use of think-aloud protocols to compare inferencing abilities in average and below-average readers. Journal of Learning Disabilities, 35(5), 436-447.

Long, D. L., y Chong, J. L. (2001). Comprehension skill and global coherence: A paradoxical picture of poor comprehenders' abilities. Journal of Experimental Psychology: Learning, Memory, and Cognition, 27, 1424-1429.

McNamara, D. S., y Kintsch, W. (1996). Learning from texts: Effects of prior knowledge and text coherence. Discourse Processes, 22, 247-288.

McNamara, D. S., Kintsch, E., Songer, N., y Kintsch, W. (1996). Are good texts always better? Interactions of text coherence, background knowledge, and levels of understanding in learning from text. Cognition and Instruction, 14, 1-43.

Means, M. L., y Voss, J. F. (1985). Star wars: A developmental study of expert and novice knowledge structures. Journal of Memory and Language, 24, 746-757.

Oakhill, J., y Yuill, N. (1986). Pronoun resolution in skilled and less-skilled comprehenders: Effects of memory load and inferential complexity. Language and Speech, 29, 25-37. 
Perfetti, C. A. (1989). There are generalized abilities and one of them is reading. En L.B. Resnick (Ed.), Knowing, learning, and instruction: Essays in honor of Robert Glaser (pp. 307-336). Hillsdale, NJ: Lawrence Erlbaum Associates, Inc.

Recht, D. R., y Leslie, L. (1988). Effect of prior knowledge on good and poor readers' memory of text. Journal of Educational Psychology, 80, 16-20.

Schmidt, R. J., Rozendal, M. S., y Greenman, G. G. (2002). Reading instruction in the inclusion classroom: Research-based practices. Remedial and Special Education, 23, 130140.

Shapiro, A.M. (2002). The Relationship between Text Structure and Reader Characteristics: A Search for Predictors of Successful Learning. Paper discussion at the Annual Meeting of the American Educational Research Association (AERA), New Orleans, April.

Spilich, G. J., Vesonder, G. T., Chiesi, H. L., y Voss, J. F. (1979). Text processing of domain-related information for individuals with high and low domain knowledge. Journal of Verbal Learning and Verbal Behavior, 18, 275-290.

van Dijk, T. (1980). Macrostructures: An interdisciplinary study of global structures in discourse, interaction, and cognition. Hillsdale, NJ: Lawrence Erlbaum Associates.

Voss, J. F., y Silfies, L. N. (1996). Learning from history text: The interaction of knowledge and comprehension skill with text structure. Cognition and Instruction, 14, 45-68.

Wood, P. (1982). The Nelson-Denny Reading Test as a predictor of college freshmen grades. Educational-and-Psychological Measurement, 42, 575-583.

Yang, C. L., Gordon, P. C., Hendrick, R., y Wu, J. T. (1999). Comprehension of referring expressions in Chinese. Language and Cognitive Processes, 14, 715-743.

Zimmer, J., Glover, J., Ronning, R., y Petersen, C. (1979). On the utility of the NelsonDenny Reading Test as a covariate in research on prose-processing. Perceptual-andMotor-Skills, 48, 641-642. 